УДК 517.958:539.374

\title{
Densification of a Viscous Porous Layer with Consideration for Elastic Effects
}

\author{
Georgiy M. Sevastyanov* \\ Institute of Machine Engineering and Metallurgy FEB RAS \\ Metallurgov, 1, Komsomolsk-on-Amur, 681005
}

Russia

Received 15.07.2016, received in revised form 23.12.2016, accepted 14.03.2017

\begin{abstract}
A viscous flow of a horizontal compressible layer under the gravity and constant external pressure is considered in this paper. It is assumed that medium motion is quasi-static and uniaxial, and the reversible and irreversible strains are finite. It is also assumed that the material is subject to the Green flow condition with coefficients depending on the material density and the plastic strain rate. The irreversible strains occur in the material at arbitrary non-zero load. The initial boundary value problem is reduced to the first-order differential equation with separable variables. This equation contains the time variable as a parameter. Evolution of the density distribution over the layer height is determined in the particular cases. An approximate analytical solution for the density in the initial phase of densification is obtained when reversible strains are negligible. The numerical solution for the density is obtained in the case of small elastic strains. These solutions are valid until a fully densified region on the underlying surface occurs. Further evolution of such region is not considered.
\end{abstract}

Keywords: compressible medium, finite strains, Almansi strain tensor, viscous flow, porosity, Green yield surface, uniaxial deformation, densification, gravity load, von Mises transformation.

DOI: 10.17516/1997-1397-2017-10-3-345-352.

\section{Introduction}

The problems of one-dimensional motions of a continuous medium are most studied in the theory of elastoviscoplasticity. However, solutions for such problems are rather difficult when geometrically nonlinear relations are taken into account. In addition, if deformations are large then separation of total strains into elastic and plastic parts is not obvious. This separation can be carried out in various ways $[1,2]$ and it is often based on the multiplicative decomposition of the deformation gradient tensor. Analytical solutions for finite reversible and irreversible strains were obtained [3-6]. In particular, the plastic flow under gravity load was considered [6]. In these papers the material is supposed to be incompressible, and the singular yield surfaces are used. The abandonment of the incompressibility condition usually complicates solution of boundary value problems. However, when certain yield surfaces are used such abandonment allows us to consider uniaxial deformation of a continuous medium. In this case, the model relations are greatly simplified. In this paper we consider the densification of a heavy flat layer of the porous material under its own weight and under the action of constant external pressure. The elliptical yield criterion, which depends on the shear stress intensity and the hydrostatic stress, was first discussed for plastically compressible materials [7]. The elliptical yield surfaces with coefficients

*akela.86@mail.ru

(C) Siberian Federal University. All rights reserved 
depending on the relative density of the material were proposed for non-continuous bodies $[8,9]$. Viscosity of materials can be taken into account in the framework of these models [10]. In this paper we assume that the yield surface has zero initial radius. The expansion of the yield surface is solely due to the viscous properties of the medium. In this case there is no elastoplastic boundary in the medium since any non-zero force causes the irreversible deformation of the material. The specified formulation of the problem may have application in modeling of foam deformation and consolidation of metal powders at high temperature. It also can be applied to model behavior of soils for which the yield strength depends on the invariants of the plastic strain rates tensor [11].

\section{Basic relationships}

For the kinematics of the finite reversible and irreversible strains we assume the following system of relations in the Euler spatial variables [12]. The irreversible (plastic) component $\boldsymbol{A}^{\boldsymbol{p}}$ of the Almansi total strain tensor $\boldsymbol{A}=\left((\boldsymbol{\nabla} \otimes \boldsymbol{u})+(\boldsymbol{\nabla} \otimes \boldsymbol{u})^{T}-(\boldsymbol{\nabla} \otimes \boldsymbol{u}) \cdot(\boldsymbol{\nabla} \otimes \boldsymbol{u})^{T}\right) / 2$ is defined by the equation

$$
\hat{A^{p}}=\varepsilon^{p}-A^{p} \cdot \varepsilon^{p}-\varepsilon^{p} \cdot A^{p}
$$

where $\hat{A^{p}}=\dot{A^{p}}-\boldsymbol{r} \cdot A^{\boldsymbol{p}}+A^{\boldsymbol{p}} \cdot \boldsymbol{r}$ is the objective derivative of the irreversible strain tensor, $\dot{A}^{\boldsymbol{p}}=\boldsymbol{A}^{\boldsymbol{p}}{ }_{t}+(\boldsymbol{v} \cdot \nabla) \boldsymbol{A}^{\boldsymbol{p}}$ is the material derivative of the irreversible strain tensor, hereinafter the subscript $t$ denotes the partial differentiation with respect to time, $\boldsymbol{v}=\boldsymbol{u}_{t}+(\boldsymbol{v} \cdot \nabla) \boldsymbol{u}$ is the velocity vector, $\boldsymbol{u}$ is the displacement vector, $\boldsymbol{r}=\boldsymbol{w}+\boldsymbol{z}$ is the antisymmetric rotation tensor; the nonlinear part $\boldsymbol{z}$ of tensor $\boldsymbol{r}$ in the general case depends on the reversible strain tensor and the strain rate tensor $[6], \boldsymbol{w}=\left((\boldsymbol{\nabla} \otimes \boldsymbol{v})^{T}-(\boldsymbol{\nabla} \otimes \boldsymbol{v})\right) / 2$ is the spin tensor, $\boldsymbol{\varepsilon}^{\boldsymbol{p}}$ is the plastic strain rate tensor, $\boldsymbol{\nabla}$ is the Hamilton operator.

The algebraic separation of the total strain into the reversible and irreversible components follows from evolution equation (1) for $\boldsymbol{A}^{\boldsymbol{p}}$ and from similar equation for $\boldsymbol{A}^{\boldsymbol{e}}$ [6]. Such separation has the form

$$
\boldsymbol{A}=\boldsymbol{A}^{\boldsymbol{e}}+\left(\boldsymbol{I}-2 \boldsymbol{A}^{\boldsymbol{e}}\right)^{1 / 2} \cdot \boldsymbol{A}^{\boldsymbol{p}} \cdot\left(\boldsymbol{I}-2 \boldsymbol{A}^{\boldsymbol{e}}\right)^{1 / 2},
$$

where $\boldsymbol{A}^{\boldsymbol{e}}$ is the reversible (elastic) component of the total strain tensor, $\boldsymbol{I}$ is the unit tensor.

The constitutive physical relation is

$$
\boldsymbol{\sigma}=\frac{\rho}{\rho_{0}} \frac{\partial W}{\partial A^{\boldsymbol{e}}} \cdot\left(\boldsymbol{I}-2 \boldsymbol{A}^{\boldsymbol{e}}\right)
$$

where $\boldsymbol{\sigma}$ is the Cauchy stress tensor, $\rho$ is the density of the medium, $\rho_{0}$ is the density of the undeformed medium, $W$ is the strain energy density (a scalar-valued function of tensor argument) which depends on the invariants of the reversible strain tensor.

The equation of equilibrium is

$$
\nabla \cdot \boldsymbol{\sigma}=-\rho \boldsymbol{g}
$$

where $\boldsymbol{g}$ is the gravity acceleration vector.

We assume that the porous material satisfies the Green flow condition [9]

$$
\Phi=\frac{\left(I_{1}^{\sigma}\right)^{2}}{9 a}+\frac{J_{2}^{\sigma}}{b}-\kappa^{2}=0
$$

where $I_{1}^{\sigma}=\operatorname{tr}(\boldsymbol{\sigma}), J_{2}^{\sigma}=\left[\operatorname{tr}\left(\boldsymbol{\sigma}^{2}\right)-\operatorname{tr}(\boldsymbol{\sigma})^{2} / 3\right] / 2$ is the quadratic invariant of the deviatoric stress tensor. Coefficients $a$ and $b$ in relation (5) are assumed to be known functions of the relative 
density $\rho / \rho_{1}$, where $\rho_{1}$ is the solid phase density. The shear yield strength of the solid phase $\kappa$ is assumed to be power function of the form $\kappa=\kappa_{0}\left(\varepsilon_{i}^{p}\right)^{\nu}$, where $\varepsilon_{i}^{p}$ is the intensity of plastic shear strain rate, $\left(\varepsilon_{i}^{p}\right)^{2} / 2=\operatorname{tr}\left(\varepsilon^{\boldsymbol{p}}\right)-\operatorname{tr}\left(\varepsilon^{\boldsymbol{p}}\right)^{2} / 3, \kappa_{0}$ and $\nu$ are some material parameters. To asymptotically transfer yield surface (5) into the von Mises cylinder it is assumed that $\lim _{\rho \rightarrow \rho_{1}} a=\infty$, $\lim _{\rho \rightarrow \rho_{1}} b=1$.

The plastic flow rule associated with yield surface (5) is

$$
\varepsilon^{p}=\lambda \frac{\partial \Phi}{\partial \boldsymbol{\sigma}}
$$

where $\lambda$ is the scalar Lagrange multiplier.

\section{Formulation of the problem}

Consider a horizontal plane layer that is initially at rest. It has thickness $h_{0}$ and the uniform density equal to $\rho_{0}$. The constant external pressure applied on the surface of the layer is equal to $P_{0}$. Let us consider the motion of the continuous medium under such conditions in order to determine evolution of the material density distribution over the layer height.

We introduce a rectangular Cartesian coordinate system $\left(X_{1}, X_{2}, X_{3}\right)$ with the origin on the underlying surface of the layer (the $X_{1}$ axis is directed downward), $\boldsymbol{X}, \boldsymbol{x}$ are the radius vectors of a point of the medium at the initial and deformed state, respectively, $\boldsymbol{X}=\boldsymbol{x}-\boldsymbol{u}$. In this case, the system of equations (1)-(6) is closed by the initial condition of zero stress-strain state and by the following boundary conditions

$$
\left.\sigma_{11}\right|_{X_{1}=0}=-\left(\rho_{0} g h_{0}+P_{0}\right),\left.\quad \sigma_{11}\right|_{X_{1}=h_{0}}=-P_{0}
$$

where $g=9.8 \mathrm{~m} / \mathrm{s}^{2}$ is the acceleration due to gravity.

We assume that density of the medium can change both reversibly and irreversibly. The yield condition (5), unlike some conditions known for a compressible medium (for example, the von Mises - Schleicher cone), allows for the uniaxial irreversible deformation. The reversible deformation is also assumed to be uniaxial.

\section{Governing equation}

In the given above formulation $\alpha_{11}=\partial u_{1} / \partial x_{1}-\left(\partial u_{1} / \partial x_{1}\right)^{2} / 2$ is the only non-zero component of the Almansi total strain tensor. The relative density of the continuous medium is governed by the strain tensor invariants: $\rho / \rho_{0}=\left(1-2 I_{1}+4 I_{2}-8 I_{3}\right)^{1 / 2}$, where $I_{1}=\operatorname{tr}(\boldsymbol{A}), 2 I_{2}=\operatorname{tr}(\boldsymbol{A})^{2}-$ $\operatorname{tr}\left(\boldsymbol{A}^{2}\right), I_{3}=\operatorname{det}(\boldsymbol{A})$. Then we have

$$
\frac{\rho}{\rho_{0}}=\sqrt{1-2 \alpha_{11}}=1-\frac{\partial u_{1}}{\partial x_{1}}
$$

Equilibrium equation (4) has the form $\partial \sigma_{11} / \partial x_{1}=-\rho g$. Using (8), we integrate this equation and obtain

$$
\sigma_{11}=-\rho_{0} g\left(C_{1}(t)+x_{1}-u_{1}\right) .
$$

From the first boundary condition (7) follows $C_{1}(t)=h_{0}+P_{0} /\left(\rho_{0} g\right)=\tilde{h}_{0}=$ const. 
Let us introduce $\omega=\tilde{h}_{0}+x_{1}-u_{1}, \omega \geqslant 0$. Taking into account $\rho / \rho_{0}=\omega_{x}$ and $\sigma_{11}=-\rho_{0} g \omega$, then equation (3) allows us to express the reversible deformation as follows

$$
\alpha_{11}^{e}=E\left(-\rho_{0} g \frac{\omega}{\omega_{x}}\right)
$$

where function $E$ is uniquely determined by the elastic potential.

In the case of uniaxial compressive strain relation $1-2 \alpha_{11}=\left(1-2 \alpha_{11}^{e}\right)\left(1-2 \alpha_{11}^{p}\right)$ follows from (2). Taking into account (8) and (10), we have

$$
1-2 \alpha_{11}^{p}=\left(\frac{\omega_{x}}{\sqrt{1-2 E\left(-\rho_{0} g \frac{\omega}{\omega_{x}}\right)}}\right)^{2} .
$$

In addition, in the case of uniaxial deformation the rotation tensor $\boldsymbol{r}$ turns out to be zero and relation (1) becomes

$$
\left(\frac{\partial}{\partial t}+v_{1} \frac{\partial}{\partial x_{1}}\right) \alpha_{11}^{p}=\varepsilon_{11}^{p}\left(1-2 \alpha_{11}^{p}\right), \quad v_{1}=\frac{\partial u_{1}}{\partial t}+v_{1} \frac{\partial u_{1}}{\partial x_{1}}=-\frac{\omega_{t}}{\omega_{x}} .
$$

Taking into account (11), we obtain

$$
\varepsilon_{11}^{p}=\left(\frac{\omega_{t}}{\omega_{x}} \frac{\partial}{\partial x_{1}}-\frac{\partial}{\partial t}\right) \ln \left(\frac{\omega_{x}}{\sqrt{1-2 E\left(-\rho_{0} g \frac{\omega}{\omega_{x}}\right)}}\right)
$$

and after some transformations we have

$$
\varepsilon_{11}^{p}=\frac{\omega_{t} \omega_{x x}-\omega_{x t} \omega_{x}}{\omega_{x}^{2}}\left(1+\rho_{0} g \frac{\omega}{\omega_{x}} \frac{E^{\prime}\left(-\rho g \frac{\omega}{\omega_{x}}\right)}{1-2 E\left(-\rho g \frac{\omega}{\omega_{x}}\right)}\right),
$$

here the prime denotes derivative with respect to argument of function $E$.

On the other hand, for arbitrary functions $a\left(\rho / \rho_{1}\right), b\left(\rho / \rho_{1}\right)$ and the power law $\kappa=\kappa_{0}\left(\varepsilon_{i}^{p}\right)^{\nu}$ in the yield surface equation (5), expression $\sigma_{11}=-F\left(\rho / \rho_{1}\right)\left|\varepsilon_{11}^{p}\right|^{\nu}$ is the result of associated flow rule (6) [13]. Taking into account (9), for $\varepsilon_{11}^{p}<0$ we have

$$
\varepsilon_{11}^{p}=-\left[\rho_{0} g \omega / F\left(\omega_{x} \frac{\rho_{0}}{\rho_{1}}\right)\right]^{1 / \nu}
$$

where function $F$ is uniquely determined by the coefficients $a\left(\rho / \rho_{1}\right)$ and $b\left(\rho / \rho_{1}\right)$.

Let us introduce new variables $\hat{t}=t\left(\rho_{1} / \rho_{0}\right)^{1 / \nu}, \hat{x}=x_{1} \rho_{0} g, \hat{\omega}=\omega g \rho_{0}^{2} / \rho_{1}$. Eliminating $\varepsilon_{11}^{p}$ from (13) and (14), we obtain the equation for the function $\hat{\omega}$

$$
\frac{\hat{\omega}_{\hat{t}} \hat{\omega}_{\hat{x} \hat{x}}-\hat{\omega}_{\hat{x} \hat{t}} \hat{\omega}_{\hat{x}}}{\hat{\omega}_{\hat{x}}^{2}}\left(1+\frac{\hat{\omega}}{\hat{\omega}_{\hat{x}}} \frac{E^{\prime}\left(-\frac{\hat{\omega}}{\hat{\omega}_{\hat{x}}}\right)}{1-2 E\left(-\frac{\hat{\omega}}{\hat{\omega}_{\hat{x}}}\right)}\right)=-\left[\frac{\hat{\omega}}{F\left(\hat{\omega}_{\hat{x}}\right)}\right]^{1 / \nu} .
$$

The von Mises transformation [14] $\zeta=\hat{t}, \eta=\hat{\omega}, \psi(\zeta, \eta)=\hat{\omega}_{\hat{x}}$ reduces the order of equation (15):

$$
\frac{\partial}{\partial \zeta}\left(\ln \left(\frac{\psi}{\sqrt{1-2 E\left(-\frac{\eta}{\psi}\right)}}\right)\right)=\left(\frac{\eta}{F(\psi)}\right)^{1 / \nu} .
$$


Differential relation (16) is an equation with separable variables in which $\eta$ appears as a parameter. This equation is valid for an arbitrary function of the internal energy density, for arbitrary coefficients $a\left(\rho / \rho_{1}\right), b\left(\rho / \rho_{1}\right)$ in flow condition (5) and for the yield strength $\kappa=\kappa_{0}\left(\varepsilon_{i}^{p}\right)^{\nu}$. In addition, pressure exerted by the layer on the underlying surface must be constant and there is no fully densified region in the material.

\section{Results}

We assume linear relationship between the yield strength and the intensity of the irreversible shear strain rate $(\nu=1)$. We specify functions $a$ and $b$ in the form $a\left(\rho / \rho_{1}\right)=4 / 3\left(\rho / \rho_{1}\right)^{4} /(1-$ $\left.\rho / \rho_{1}\right), b\left(\rho / \rho_{1}\right)=\left(\rho / \rho_{1}\right)^{3}[15]$. Using [13],one can show that in this case function $F$ in (16) has the form

$$
F(\psi)=\frac{4 \kappa_{0}}{3}\left(\frac{\psi^{3}}{1-\psi}\right)^{1 / 2} .
$$

Let us consider some variants of the formulated problem.

I. Ideal flow. If reversible change in the density of the material is insignificant, integration of (16) with respect to $\zeta$ gives

$$
\int \psi^{-1} F(\psi)^{1 / \nu} d \psi=\eta^{1 / \nu} \zeta+C_{0}(\eta)
$$

Taking into account (17), for $\nu=1$ we have

$$
-4 / 3 \kappa_{0}(\sqrt{\psi(1-\psi)}+\arccos \sqrt{\psi})=\eta \zeta+C_{0}(\eta)
$$

$C_{0}(\eta)=-4 / 3 \kappa_{0}\left(\sqrt{\psi_{0}\left(1-\psi_{0}\right)}+\arccos \sqrt{\psi_{0}}\right)=$ const, where $\psi_{0}=\rho_{0} / \rho_{1}$, follows from initial conditions $\zeta=0, \psi=\psi_{0}$.

Relation (18) is an implicit expression for $\omega_{x}$ in term of $\omega$. Time $t$ is included in (18) as a parameter. Solution of equation (18) with respect to $\omega$ contains an arbitrary function of time. To determine this function one should use the first boundary condition (7) in the form of $\left.\omega\right|_{x_{1}=0}=\tilde{h}_{0}$.

When $\psi_{0} \leqslant \psi<<1$ one can assume that $\sqrt{\psi(1-\psi)}+\arccos \sqrt{\psi} \approx \pi / 2-2 / 3 \psi^{3 / 2}$. Then relation (18) can be approximately expressed as $\eta \zeta=8 / 9 \kappa_{0}\left(\psi^{3 / 2}-\psi_{0}^{3 / 2}\right)$ or

$$
\omega_{x}=(1+k \omega t)^{2 / 3}, \quad k=\frac{9 \rho_{0} g}{8 \kappa_{0} \psi_{0}^{3 / 2}} .
$$

Then

$$
k \omega t=-1+\left(\frac{k x_{1} t}{3}+f(t)\right)^{3}, \quad f(t)=\left(1+k \tilde{h}_{0} t\right)^{1 / 3}
$$

and the density distribution satisfies the following relation

$$
\frac{\rho}{\rho_{0}}=\left(\frac{k x_{1} t}{3}+f(t)\right)^{2} .
$$

Analytical solution (19), (20) is valid only if the state of the material is far from the state of full consolidation. 
II. Small elastic strains. If the elastic strain $\alpha_{11}^{e}=E\left(\sigma_{11} \rho_{0} / \rho\right)=E(-\eta / \psi)$ is small then $-\ln (1-2 E(-\eta / \psi)) / 2 \approx E(-\eta / \psi)$. The expansion of $E$ in powers of $(-\eta / \psi)$ does not contain constant term because $E(0)=0$. Let us take into account only linear term of the series with coefficient $\chi_{1}$. Considering (17), we integrate (16) for $\nu=1$ and obtain

$$
\frac{4 \kappa_{0}}{3} \int\left(\frac{1}{\psi}+\frac{\chi_{1} \eta}{\psi^{2}}\right) \frac{\psi^{3 / 2}}{\sqrt{1-\psi}} d \psi=\eta \zeta+C_{1}(\eta)
$$

where function $C_{1}(\eta)$ is uniquely identified by initial conditions, as in the previous case, but it is not a constant. Coefficient $\chi_{1}$ is determined by elastic moduli of the material. After some mathematical treatment we have

$$
\omega=\frac{\arccos \sqrt{\psi_{0}}-\arccos \sqrt{\psi_{0} \omega_{x}}+\sqrt{\psi_{0}\left(1-\psi_{0}\right)}-\sqrt{\psi_{0} \omega_{x}\left(1-\psi_{0} \omega_{x}\right)}}{\rho_{0} g\left(t / \kappa_{0}-2 \psi_{0} \chi_{1}\left(\arccos \sqrt{\psi_{0}}-\arccos \sqrt{\psi_{0} \omega_{x}}\right)\right)} .
$$

Relation (21) and the second boundary condition (7) in the form $\omega=\tilde{h}_{0}$ on the surface $x_{1}=0$ uniquely determine function $\omega$ at any moment $t>0$ and, consequently, all unknown variables of the problem. The current position of the free surface of the layer is determined by equality $\omega=P_{0} /\left(\rho_{0} g\right)$. The results of numerical solution of equation (21) in the form of the density distribution over the current layer height $h$ at different time points are shown at Fig. 1.

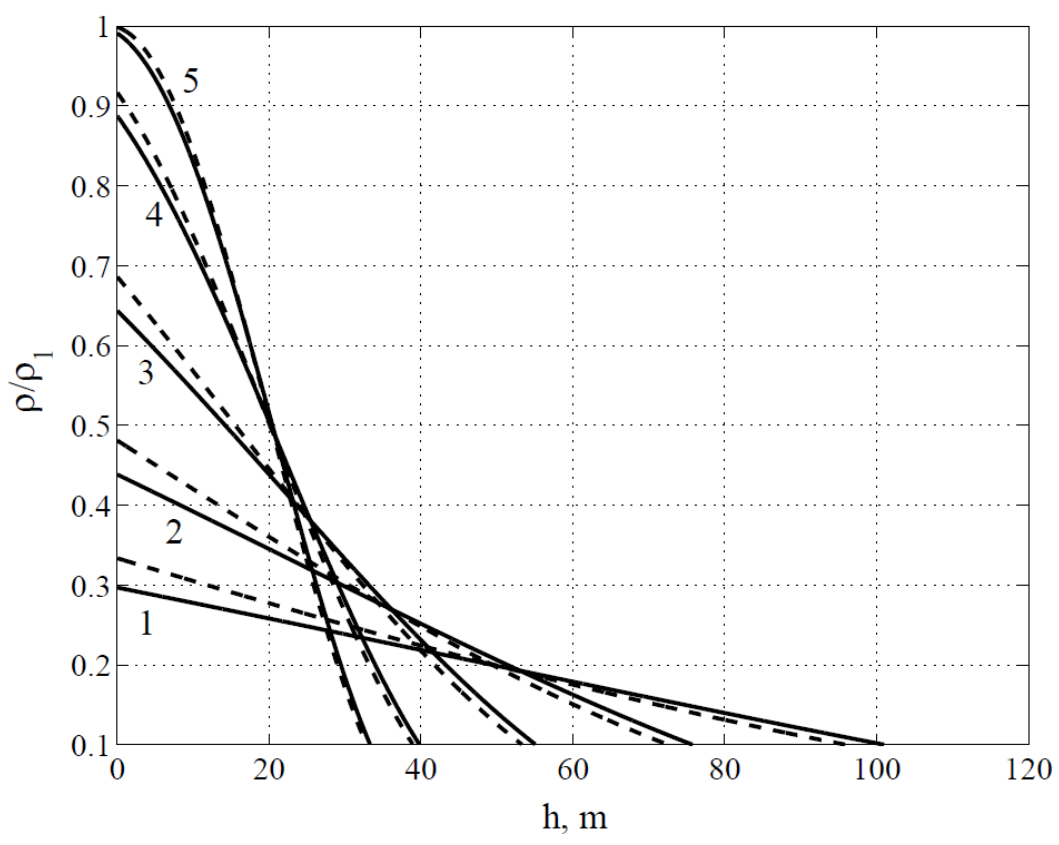

Fig. 1. The distribution of the relative density over the current layer height at different time points $t: 1-t=250 \mathrm{~s}, 2-t=500 \mathrm{~s}, 3-t=1000 \mathrm{~s}, 4-t=2000 \mathrm{~s}, 5-t=3000 \mathrm{~s}$

Dashed lines correspond to the density distribution at the same time points without taking into account the elastic deformation component $\left(\chi_{1}=0\right)$.

The following parameters of the problem are used: $\rho_{0}=250 \mathrm{~kg} / \mathrm{m}^{3}, \rho_{1}=2500 \mathrm{~kg} / \mathrm{m}^{3}$, $h_{0}=200 \mathrm{~m}, P_{0}=0, \kappa_{0}=10^{9} \mathrm{~Pa} \cdot s, \chi_{1}=10^{-6} \mathrm{~Pa}^{-1}$. The elastic moduli and hence the stiffness coefficient $\chi_{1}$ are related to the material with the initial density $\rho_{0}$. Coefficient $\kappa_{0}$ is related to the solid material with the limit density $\rho_{1}$. 
The obtained solutions exist until the material density at the underlying surface $x_{1}=0$ reaches the limit value $\rho_{1}$. From equation (21) follows that it will occur at the time moment

$$
t_{1}=\kappa_{0}\left[\left(\arccos \sqrt{\psi_{0}}+\sqrt{\psi_{0}\left(1-\psi_{0}\right)}\right) /\left(\rho_{0} g \tilde{h}_{0}\right)+2 \psi_{0} \chi_{1} \arccos \sqrt{\psi_{0}}\right]
$$

For the parameter set given above we have $t_{1}=3.41 \cdot 10^{3} \mathrm{~s}$. If elastic properties of the material are not taken into account then $t_{1}=3.16 \cdot 10^{3} \mathrm{~s}$. The influence of the elastic properties becomes noticeable when initial thickness of the layer is large enough or for significant external pressure.

Remark. Let us consider the expansion of the quantity $-\ln (1-2 E(-\eta / \psi)) / 2$ in equation (16). The expansion can include an arbitrary number of terms, i. e., the requirement of smallness of the reversible strains is not essential. In this case, taking into account (17), solution $\eta=\eta(\zeta, \psi)$ can be found for an arbitrary exponent $\nu>0$ in the form of combination of incomplete Beta-functions by virtue of the equality $\int \psi^{-n}\left(\psi^{3} /(1-\psi)\right)^{1 /(2 \nu)} d \psi=$ $B(\psi ; 1-n+3 /(2 \nu) ; 1-1 /(2 \nu))$.

\section{References}

[1] V.A.Palmov, E.Stein, Comparison of different decompositions of strain using exact solution of elastoplasticity for large strains, Proceedings of SPIE . The International Society for Optical Engineering, 5127(2003), 159-172.

[2] A.I.Golovanov, Kinematics of finite elastoplastic deformations, Russian Mathematics, 54(2010), no. 7, 12-25.

[3] L.V.Kovtanyuk, G.L.Panchenko, Straight flow in an elastoviscoplastic cylindrical layer with possible two-sided slip, Mechanics of Solids, 51(2016), no. 2, 197-205.

[4] A.A.Burenin, L.V.Kovtanyuk, The development and deceleration of the flow of an elastoplastic medium in a cylindrical tube, Journal of Applied Mathematics and Mechanics, $77(2013)$, no. 5, 566-572.

[5] A.A.Burenin, L.V.Kovtanyuk, A.L.Mazelis, Development of a rectilinear axisymmetric viscoplastic flow and elastic aftereffect after its stop, Journal of Applied Mechanics and Technical Physics, 51(2010), no. 2, 261-268.

[6] A.A.Burenin, L.V.Kovtanyuk, On elastic strains and a viscoplastic flow in a heavy layer placed on an inclined plane, Mechanics of Solids, 45(2010), no. 2, 284-294.

[7] A.E.Green, P.M.Naghdi, A comment on Drucker's postulate in the theory of plasticity, Acta Mechanica, 1(1965), no. 4, 334-338.

[8] H.A.Kuhn, C.L.Downey, Deformation Characteristics and Plasticity Theory of Sintered Powder Materials, International Journal of Powder Metallurgy, 7(1971), no.1, 15-25.

[9] R.J.Green, A plasticity theory for porous solids, International Journal of Mechanical Sciences, 14(1972), no. 4, 215-224. 
[10] A.F.Fedotov, P.I.Krasnoshchekov, Model of plastic deformation of powder materials taking account of the proportion of contact volume, Powder Metallurgy and Metal Ceramics, 44(2005), no. 9-10, 420-425.

[11] I.Towhata, T.T.L.Anh, R.Motamed, V.Sesov, Rate dependent nature of liquefied sand undergoing large flow deformation and its interaction with group pile foundation, Proceedings of the International Offshore and Polar Engineering Conference, 2009, 1-8.

[12] A.A.Burenin, G.I.Bykovtsev, L.V.Kovtanyuk, A simple model of finite strain in an elastoplastic medium, Doklady Physics, 41(1996), no. 3, 127-129.

[13] S.E.Aleksandrov, B.A.Druyanov, Pressing of a compact plastic material, Journal of Applied Mechanics and Technical Physics, 31(1990), no. 1, 108-113.

[14] A.D.Polyanin, Von Mises- and Crocco-type transformations: order reduction of nonlinear equations, RF-pairs, and Backlund transformations, Doklady Mathematics, 81(2010), no. 1, $131-136$.

[15] V.V.Skorokhod, L.I.Tuchinskii, Condition of plasticity of porous bodies, Soviet Powder Metallurgy and Metal Ceramics, 17(1979), no. 11, 880-883.

\section{Вязкое уплотнение тяжелого пористого слоя с учетом упругих эффектов}

\section{Георгий М. Севастьянов}

Институт машинной инженерии и металлургии ДВО РАН Металлургов, 1, Комсомольск-на-Амуре, 681005

Россия

В квазистатическом приближении рассмотрено вязкое течение горизонтального слоя сжимаемого материала из состояния покоя под действием собственного веса и постоянного внешнего давления. Одноосные обратимые и необратимые деформации полагаются большими. Поверхность текучести принята в форме Грина с коэффициентами, зависящими от плотности материала и скоростей пластических деформачий. При этом полагаетсл, что необратимые деформачии возникают в материале при любой отличной от нуля нагрузке. Начально-краевая задача сведена к интегрированию дифференциального уравнения первого порядка с разделяющимися переменными, в которое время входит в качестве параметра. В частных случаях определена эволюиия распределенной по высоте слоя плотности материала. А именно в случае пренебрежимых обратимых деформаций для плотности в началъной фазе уплотнения получено приближенное аналитическое решение; в случае малых упругих дебормаций построено численное решение. Указанные решения справедливы до момента зарождения на подстилающей поверхности слоя полностью уплотненной области, дальнейшая эволючия которой не рассматривается.

Ключевые слова: сюсимаемая среда, конечные деформации, тензор Алъманси, вязкое течение, пористость, поверхность текучести Грина, одноосная деформачия, уплотнение, сила тяжести, преобразование Мизеса. 